Eva Bayer-Fluckiger · Grégory Berhuy · Pascale Chuard-Koulmann

\title{
CM-fields and skew-symmetric matrices
}

Received: 3 September 2002 / Revised version: 7 December 2003

Published online: 21 May 2004

\begin{abstract}
Cohen and Odoni prove that every $\mathrm{CM}$-field can be generated by an eigenvalue of some skew-symmetric matrix with rational coefficients. It is natural to ask for the minimal dimension of such a matrix. They show that every CM-field of degree $2 n$ is generated by an eigenvalue of a skew-symmetric matrix over $\mathbf{Q}$ of dimension at most $4 n+2$. The aim of the present paper is to improve this bound.
\end{abstract}

\section{Introduction}

In [4], Cohen and Odoni show that every CM-field is generated by an eigenvalue of some skew-symmetric matrix with rational coefficients. They also ask for the minimal dimension of such a matrix. Using a result of Bender [2], they prove that every CM-field of degree $2 n$ is generated by an eigenvalue of a skew-symmetric matrix over $\mathbf{Q}$ of dimension at most $4 n+2$. The aim of the present paper is to show that this bound can be improved to $2 n+3$ if $n \equiv 1(\bmod 4)$, to $2 n+1$ if $n \equiv 3(\bmod 4)$, and to $2 n+4$ if $n$ is even.

We start with a general discussion of skew-symmetric matrices of given rank and a given eigenvalue. These conditions imply some restrictions on the characteristic polynomial of the matrix. Hence it is natural to study skew-symmetric matrices having a given characteristic polynomial. It is easy to see that the characteristic polynomial of a skew-symmetric matrix is even or odd. Conversely, let $P \in \mathbf{Q}[X]$ be a monic polynomial of degree $m$ such that $P(-X)=(-1)^{m} P(X)$. Let $A=\mathbf{Q}[X] /(P)$, and let $\sigma: A \rightarrow A$ be the $\mathbf{Q}$-linear involution induced by $X \mapsto-X$. We show that there exists a skew-symmetric matrix over $\mathbf{Q}$ with characteristic polynomial $P$ if and only if the $m$-dimensional unit form satisfies a certain invariance relation with respect to $(A, \sigma)$ (see $\S 1)$. This is just a more conceptual formulation of a well-known method of finding skew-symmetric (symmetric, orthogonal,...) matrices having a given eigenvalue (see for instance [2], [1]). After proving some preliminary results in $\S 2$, we apply this method in $\S 3$.

E. Bayer-Fluckiger: Mathématiques, Ecole Polytechnique Fédérale de Lausanne, 1015 Lausanne, Switzerland. e-mail: eva.bayer@epfl.ch

G. Berhuy: Department of Mathematics, University of British Columbia, Vancouver BC, V6S 1C2, Canada. e-mail: berhuy@ math.ubc.ca

P. Chuard-Koulmann: Institut de Mathématiques, Université de Neuchâtel, rue Emile Argand 11, 2007 Neuchâtel, Switzerland. e-mail: pascale.chuard@unine.ch 


\section{Skew-symmetric matrices and adjoint involutions}

Let $k$ be a field of characteristic $\neq 2$.

\section{Adjoint involutions}

Let $A$ be a commutative $k$-algebra, and let $\tau: A \rightarrow A$ be a $k$-linear involution. Let $q: A \times A \rightarrow k$ be a symmetric bilinear form defined over the $k$-vector space $A$. We say that the algebra with involution $(A, \tau)$ is adjoint to $q$ if

$$
q(x y, z)=q(y, \tau(x) z)
$$

for all $x, y, z \in A$. If $A$ is given and if $(A, \tau)$ is adjoint to $q$, then we shall use the notation $\tau=\tau_{q}$.

The following remark will often be implicitly used in the proofs:

Remark. Assume that $(A, \tau) \simeq\left(A^{\prime}, \tau^{\prime}\right) \times\left(A^{\prime \prime}, \tau^{\prime \prime}\right)$, for some algebras with involution $\left(A^{\prime}, \tau^{\prime}\right)$ and $\left(A^{\prime \prime}, \tau^{\prime \prime}\right)$. Let $q: A \times A \rightarrow k$ such that $\tau=\tau_{q}$. Then $q$ is isomorphic to a direct sum of symmetric bilinear forms $q^{\prime}: A^{\prime} \times A^{\prime} \rightarrow k$ and $q^{\prime \prime}: A^{\prime \prime} \times A^{\prime \prime} \rightarrow k$ with $\tau^{\prime}=\tau_{q^{\prime}}$ and $\tau^{\prime \prime}=\tau_{q^{\prime \prime}}$.

Recall that $A$ is an étale algebra if it is isomorphic to a product of a finite number of separable field extensions of finite degree of $k$. Let $\operatorname{Tr}: A \rightarrow k$ be the trace map. Then $A$ is étale if and only if the symmetric bilinear form $\operatorname{Tr}: A \times A \rightarrow k$, given by $(x, y) \mapsto \operatorname{Tr}(x y)$, is non-degenerate.

Proposition 1.1. Suppose that $A$ is an étale algebra. Then the following are equivalent :

(a) $\tau=\tau_{q}$;

(b) there exists $\alpha \in A$ such that $\tau(\alpha)=\alpha$, and that $q(x, y)=\operatorname{Tr}(\alpha x \tau(y))$.

Moreover, $q$ is non-degenerate if and only if $\alpha \in A^{*}$.

Proof. This is well-known, and follows from the fact that $\operatorname{Tr}: A \times A \rightarrow k$ is a non-degenerate symmetric bilinear form.

Let us denote by $q_{(A, \tau, \alpha)}$ the symmetric bilinear form $A \times A \rightarrow k$ given by $(x, y) \mapsto \operatorname{Tr}(\alpha x \tau(y))$. If the involution is trivial (that is, $\tau$ is the identity) then we set $q_{(A, \tau, \alpha)}=q_{(A, \alpha)}$. Note that $q_{(A, 1)}$ is the usual trace form of the algebra $A$.

\section{Skew-symmetric matrices}

Let $P \in k[X]$ be a monic polynomial of degree $m$ with $P(-X)=(-1)^{m} P(X)$ (that is, $P$ is even or odd). It is natural to ask whether $P$ is the characteristic polynomial of some skew-symmetric matrix over $k$. Set $A=k[X] /(P)$, and let $\tau: A \rightarrow A$ be the $k$-linear involution induced by $\tau(X)=-X$. Let us denote by $m .<1>$ the $m$-dimensional unit form. 
Proposition 1.2. Suppose that $P$ is separable. Then there exists a skew-symmetric matrix with coefficients in $k$ having characteristic polynomial $P$ if and only if $(A, \tau)$ is adjoint to $m .<1>$.

Proof. We recall the proof for the convenience of the reader.

Let $V$ be an $m$-dimensional $k$-vector space, and let $\left(e_{1}, \ldots, e_{m}\right)$ a basis of $V$. Let $b_{0}: V \times V \rightarrow k$ be given by $b_{0}\left(e_{i}, e_{j}\right)=\delta_{i, j}$.

Let $M \in M_{m}(k)$ such that $M^{t}=-M$, and that the characteristic polynomial of $M$ is $P$. Let $\mu: V \rightarrow V$ be the endomorphism given by the matrix $M$ in this basis. Let us endow $V$ with the $A$-module structure induced by $\mu$ (that is, the action of $X$ is given by $\mu$ ). Then $V$ is a free $A$-module of rank one. As $M$ is skew-symmetric, we have $b_{0}(\mu x, y)=b_{0}(x, \tau(\mu)(y))$ for all $x, y \in V$. This proves that $\tau=\tau_{b_{0}}$.

Conversely, suppose that $\tau=\tau_{b_{0}}$. Let us denote by $\mu: A \rightarrow A$ the endomorphism given by multiplication by the image of $X$ in $A$. Then the characteristic polynomial of $\mu$ is $P$. As $\tau=\tau_{b_{0}}$, we have $b_{0}(\mu x, y)=b_{0}(x, \tau(\mu) y)=-b_{0}(x, \mu y)$. Then $M^{t}=-M$. This concludes the proof of the proposition.

\section{Adjoint involutions and CM-fields}

\section{Invariants of symmetric bilinear forms}

Let $V$ be a finite dimensional $k$-vector space, and let $q: V \times V \rightarrow k$ be a nondegenerate symmetric bilinear form. Set $m=\operatorname{dim}(V)$. We recall the definition of some classical invariants. For more details, see for instance [6].

Determinant. The determinant of $q$, denoted by $\operatorname{det}(q)$, is by definition the determinant of the matrix of $q$ in some $k$-basis of $V$, considered as an element of $k^{*} / k^{* 2}$.

Recall that every symmetric bilinear form can be diagonalised. In other words, there exist $a_{1}, \ldots, a_{m}$ such that $q \simeq<a_{1}, \ldots, a_{m}>$.

Hasse-Witt invariant. Let $q \simeq<a_{1}, \ldots, a_{m}>$. The Hasse-Witt invariant of $q$ is by definition

$$
w_{2}(q)=\Sigma_{i<j}\left(a_{i}, a_{j}\right) \in \operatorname{Br}_{2}(k),
$$

where $\left(a_{i}, a_{j}\right)$ is the quaternion algebra determined by $a_{i}, a_{j}$ and $\operatorname{Br}_{2}(k)$ is the subgroup of elements of order one or two of the Brauer group of $k$, written additively.

Signature. Let $v$ be an ordering of $k$, and let $k_{v}$ be a real closure of $k$ at $v$. Then over $k_{v}$, the symmetric bilinear form $q$ is isomorphic to a diagonal form $<1, \ldots, 1,-1, \ldots,-1>$. Let us denote by $r$ the number of 1 's and by $s$ the number of -1 's in this diagonalisation. Then the signature of $q$ at $v$ is by definition $\operatorname{sign}_{v}(q)=r-s$. 


\section{Adjoint involutions over separable field extensions}

Let $K$ be a separable extension of $k$ of finite degree. Let $\sigma: K \rightarrow K$ be a non-trivial $k$-linear involution. Let $F$ be the fixed field of this involution, that is $F=\{x \in K \mid \sigma(x)=x\}$. Then $K$ is a quadratic extension of $F$. Let $\theta \in F^{*}$ such that $K=F(\sqrt{\theta})$.

Lemma 2.1. We have

$$
q_{(K, \sigma, \alpha)} \simeq<2>\otimes\left[q_{(F, \alpha)} \oplus q_{(F,-\alpha \theta)}\right] .
$$

Proof. This follows from the orthogonal decomposition $K=F \oplus F \sqrt{\theta}$.

Proposition 2.2. We have

(i) $\operatorname{det} q_{(K, \sigma, \alpha)}=\mathrm{N}_{F / k}(-\theta) \in k^{*} / k^{* 2}$.

(ii) $\operatorname{sign}_{v} q_{(K, \sigma, \alpha)}=\Sigma_{w}\left(1-\operatorname{sgn}_{w}(\theta)\right) \operatorname{sgn}_{w}(\alpha)$, where the sum is taken over all orderings $w$ of $F$ extending $v$, and $\operatorname{sgn}_{w}(x)$ is the sign of $x$ at $w$.

Proof. Apply lemma 2.1 and the formulas given in theorems 2.5.12. and 3.4.5. of [6].

\section{CM-fields}

Let $K$ be a CM-field. By definition $K$ is a totally imaginary algebraic number field having a non-trivial $\mathbf{Q}$-linear involution $\sigma: K \rightarrow K$, and the fixed field $F$ of this involution is totally real. Set $n=[F: \mathbf{Q}]$. Then $[K: \mathbf{Q}]=2 n$. It is well-known that there exists a totally negative element $\theta \in F^{*}$ such that $K=F(\sqrt{\theta}$ ) (see for instance [4]). Note that the involution $\sigma$ is given by $\sigma(\sqrt{\theta})=-\sqrt{\theta}$.

We denote by $n .<1>$ the $n$-dimensional unit form. Let $d_{F}$ be the discriminant of the field $F$, that is the determinant of $q_{(F, 1)}$. It is well-known that

$$
d_{F}=\prod_{i<j}\left(\gamma_{i}-\gamma_{j}\right)^{2} \bmod \mathbf{Q}^{* 2},
$$

where the $\gamma_{i}$ 's denote the conjugates of a primitive element of $F$.

Proposition 2.3. Let $K$ be a $C M$-field of degree $2 n$, with $n$ odd. Let $\alpha \in F^{*}$ be totally positive. Then we have

$$
q_{(K, \sigma, \alpha)} \simeq<\mathrm{N}_{F / \mathbf{Q}}\left(2 \alpha d_{F}\right)>\otimes<1,-\mathrm{N}_{F / \mathbf{Q}}(\theta)>\otimes(n .<1>) .
$$

Proof. Note that $\alpha$ and $-\theta \alpha$ are both totally positive. Hence by lemma 2.1. it suffices to check that for any totally positive $\gamma \in F^{*}$, we have

$$
q_{(F, \gamma)} \simeq<d_{F} \mathrm{~N}_{F / \mathbf{Q}}(\gamma)>\otimes(n .<1>) .
$$

Set $b_{\gamma}=<d_{F} \mathrm{~N}_{F / \mathbf{Q}}(\gamma)>\otimes(n .<1>)$. The forms $q_{(F, \gamma)}$ and $b_{\gamma}$ have equal dimensions and determinants. As $F$ is totally real, $d_{F}$ is positive. Since $\gamma$ is totally positive, $\mathrm{N}_{F / \mathbf{Q}}(\gamma)$ is also positive. Therefore $\operatorname{sign}\left(b_{\gamma}\right)=n$. We also have 
$\operatorname{sign} q_{(F, \gamma)}=n$, hence $(F, \gamma)$ and $b_{\gamma}$ have equal signatures. This implies that $(F, \gamma)$ and $b_{\gamma}$ are isomorphic over $\mathbf{R}$. In particular, over $\mathbf{R}$ the forms $q_{(F, \gamma)}$ and $b_{\gamma}$ have equal Hasse-Witt invariants. Let us check that $q_{(F, \gamma)}$ and $b_{\gamma}$ also have equal Hasse-Witt invariants over the $p$-adic numbers $\mathbf{Q}_{p}$ for all prime numbers $p$. When $p \neq 2$ this follows from [5]. By the product formula, this holds also for $p=2$. Hence $q_{(F, \gamma)}$ and $b_{\gamma}$ have equal dimensions, determinants, signatures and Hasse-Witt invariants. By the Hasse-Minkowski theorem, they are isomorphic (see for instance [6], Chap. 6).

\section{Skew-symmetric matrices associated with CM-fields}

Let $K$ be a CM-field of degree $2 n$. We keep the notation of $\S 2$. In particular, $\theta \in F^{*}$ is a totally negative element such that $K=F(\sqrt{\theta})$. Let $f \in \mathbf{Q}[X]$ be the minimal polynomial of $\theta$.

Cohen and Odoni (cf. [4]) have shown that there exist skew-symmetric matrices over $\mathbf{Q}$ with eigenvalue $\sqrt{\theta}$. In this section, we give an upper bound for the minimal dimension of such a matrix. We deal separately with the cases $n$ odd and $n$ even.

Theorem 3.1. Suppose that $n$ is odd. Then there exists a skew-symmetric matrix over $\mathbf{Q}$ of dimension $2 n+3$ with eigenvalue $\sqrt{\theta}$.

This theorem is a consequence of prop. 3.2.- 3.6. below.

Proposition 3.2. Suppose that $n$ is odd. Then $\sqrt{\theta}$ is an eigenvalue of a skew-symmetric matrix of dimension $2 n$ if and only if $-\mathrm{N}_{F / \mathbf{Q}}(\theta) \in \mathbf{Q}^{* 2}$.

Proof. There exists a skew-symmetric matrix over $\mathbf{Q}$ of dimension $2 n$ with eigenvalue $\sqrt{\theta}$ if and only if there exists a skew-symmetric matrix over $\mathbf{Q}$ of characteristic polynomial $f$. By prop. 1.2., this holds if and only if $(K, \sigma)$ is adjoint to the $2 n-$ dimensional unit form $2 n$. $<1>$. Using prop. 1.1., we see that this is equivalent with the existence of an $\alpha \in F^{*}$ such that $q_{(K, \sigma, \alpha)} \simeq 2 n$. $<1>$. Comparing determinants, we see that this implies that $-\mathrm{N}_{F / \mathbf{Q}}(\theta) \in \mathbf{Q}^{* 2}$. Conversely, suppose that $-\mathrm{N}_{F / \mathbf{Q}}(\theta) \in \mathbf{Q}^{* 2}$. Set $\alpha=2 d_{F}$. This is a positive rational number. By prop. 2.3., we get $q_{(K, \sigma, \alpha)} \simeq 2 n$. $<1>$. This concludes the proof of the proposition.

Proposition 3.3. Suppose that $n \equiv 3(\bmod 4)$. Then $\sqrt{\theta}$ is the eigenvalue of a skew-symmetric matrix of dimension $2 n+1$.

Note that the two previous propositions show that $2 n+1$ is the best possible bound when $n \equiv 3(\bmod 4)$.

The following lemma is well-known :

Lemma 3.4. For any positive rational number a, we have

$$
<a>\otimes<1,1,1,1>\simeq<1,1,1,1>.
$$


Proof. By Lagrange's theorem, every positive rational number is a sum of four squares. Hence any such number $a$ is represented by $<1,1,1,1>$. On the other hand, this form is multiplicative (see for instance [6], chap. 2). This implies the desired statement.

Proof of prop. 3.3. Let $P(X)=X f(X)$. Notice that $\sqrt{\theta}$ is the eigenvalue of a skew-symmetric matrix over $\mathbf{Q}$ of dimension $2 n+1$ if and only if there exists a skew-symmetric matrix over $\mathbf{Q}$ with characteristic polynomial $P$. By prop. 1.1. and 1.2., this is the case if and only if there exist $\alpha \in F^{*}$ and $a \in \mathbf{Q}^{*}$ such that $q_{(K, \sigma, \alpha)} \oplus<a>\simeq(2 n+1) .<1>$.

By prop. 2.3. we have

$$
q_{(K, \sigma, \alpha)} \simeq<\mathrm{N}_{F / \mathbf{Q}}\left(2 \alpha d_{F}\right)>\otimes<1,-\mathrm{N}_{F / \mathbf{Q}}(\theta)>\otimes(n .<1>) .
$$

Set $\alpha=2 d_{F}$. Then

$$
q_{(K, \sigma, \alpha)} \oplus<-\mathrm{N}_{F / \mathbf{Q}}(\theta)>\simeq<-\mathrm{N}_{F / \mathbf{Q}}(\theta)>\otimes((n+1) .<1>) \oplus n .<1>.
$$

As $n+1 \equiv 0(\bmod 4)$, by lemma 3.4. we have

$$
-\mathrm{N}_{F / \mathbf{Q}}(\theta) \otimes((n+1) .<1>) \simeq(n+1) .<1>.
$$

Therefore

$$
q_{(K, \sigma, \alpha)} \oplus<-\mathrm{N}_{F / \mathbf{Q}}(\theta)>\simeq(2 n+1) .<1>.
$$

Hence prop. 3.3. is proved.

Proposition 3.5. Suppose that $n \equiv 1(\bmod 4)$. Then $\sqrt{\theta}$ is an eigenvalue of a skew-symmetric matrix of dimension $2 n+3$.

Proof. Let $d \in \mathbf{Q}^{*}$ be a sum of two squares, and suppose that $d \neq-\mathrm{N}_{K / \mathbf{Q}}(\theta)$ in $\mathbf{Q}^{*} / \mathbf{Q}^{* 2}$. Set $P(X)=X\left(X^{2}+d\right) f(X)$. Then $P$ is a separable polynomial (this is clear if $n>1$, and this is by choice of $d$ if $n=1)$. Let $E=\mathbf{Q}[X] /(P)$, and let $\tau: E \rightarrow E$ be the $\mathbf{Q}-$ linear involution induced by $X \mapsto-X$. By prop. 1.2., it suffices to show that $(E, \tau)$ is adjoint to the $(2 n+3)$-dimensional unit form. It is easy to see that if $(E, \tau)$ is adjoint to some non-degenerate symmetric bilinear form $q$ if and only if $q \simeq q_{(K, \sigma, \alpha)} \oplus<2 a, 2 a d>\oplus<b>$ for some $\alpha \in F^{*}, a, b \in \mathbf{Q}^{*}$. Hence by prop. 1.1. it is enough to show that there exist $\alpha \in F^{*}, a, b \in \mathbf{Q}^{*}$, such that

$$
q_{(K, \sigma, \alpha)} \oplus<2 a, 2 a d>\oplus<b>\simeq(2 n+3) .<1>.
$$

Set $\alpha=2 d_{K}, a=-2 \mathrm{~N}_{F / \mathbf{Q}}(\theta)$ and $b=-d \mathrm{~N}_{F / \mathbf{Q}}(\theta)$. Then we have, using prop. 3.3.

$$
\begin{gathered}
q_{(K, \sigma, \alpha)} \oplus<2 a, 2 a d>\oplus<b>\simeq \\
<1,-\mathrm{N}_{F / \mathbf{Q}}(\theta)>\otimes(n .<1>) \oplus<-\mathrm{N}_{F / \mathbf{Q}}(\theta)>\otimes<1, d, d>.
\end{gathered}
$$

As $d$ is a sum of two squares, $<d, d>\simeq<1,1>$. Hence the above form is isomorphic to $n .<1>\oplus<-\mathrm{N}_{F / Q}(\theta)>\otimes(n+3) .<1>$. As $n+3$ is divisible by 4 , we have $<-\mathrm{N}_{F / \mathbf{Q}}(\theta)>\otimes(n+3) .<1>\simeq(n+3) .<1>$. Hence we get the form $(2 n+3) .<1>$, as claimed. 
As shown in prop. 3.6. below, it is sometimes possible to get a better bound:

Proposition 3.6. Suppose that $n \equiv 1(\bmod 4)$. Then the following are equivalent:

1. $\sqrt{\theta}$ is an eigenvalue of a skew-symmetric matrix of dimension $2 n+1$;

2. $-\mathrm{N}_{F / \mathbf{Q}}(\theta)$ is a sum of three squares in $\mathbf{Q}$.

Proof. As seen in the proof of prop. 3.2., condition (i) holds if and only if

$$
q_{(K, \sigma, \alpha)} \oplus<a>\simeq(2 n+1) .<1>
$$

for some $\alpha \in F^{*}, a \in \mathbf{Q}^{*}$. Note that if this isomorphism holds, then by comparing determinants we get $a=-\mathrm{N}_{F / \mathbf{Q}}(\theta) \in \mathbf{Q}^{*} / \mathbf{Q}^{* 2}$. Hence we can assume that $a=-\mathrm{N}_{F / \mathbf{Q}}(\theta)$. Comparing signatures, we get that $\alpha$ is totally positive. Set $\beta=2 \alpha d_{F}$. Then by prop. 2.3.,

$$
\begin{gathered}
q_{\left(K, \sigma, q_{\alpha}\right)} \oplus<-\mathrm{N}_{F / \mathbf{Q}}(\theta)>\simeq \\
<\mathrm{N}_{F / \mathbf{Q}}(\beta)>\otimes<1,-\mathrm{N}_{F / \mathbf{Q}}(\theta)>\otimes(n .<1>) \oplus<-\mathrm{N}_{F / \mathbf{Q}}(\theta)>.
\end{gathered}
$$

This form is isomorphic to the $(2 n+1)$-dimensional unit form if and only if

$$
<\mathrm{N}_{F / \mathbf{Q}}(\beta),-\mathrm{N}_{F / \mathbf{Q}}(\theta \beta),-\mathrm{N}_{F / \mathbf{Q}}(\theta)>\simeq<1,1,1>
$$

(use lemma 3.4. and Witt cancellation). We claim that this happens if and only if $-\mathrm{N}_{F / \mathbf{Q}}(\theta)$ is a sum of three squares. The necessity of this condition is clear. Conversely, suppose that $-\mathrm{N}_{F / \mathbf{Q}}(\theta)$ is a sum of three squares. Then there exists a positive $b \in \mathbf{Q}^{*}$ such that

$$
<1,1,1>\simeq<-\mathrm{N}_{F / \mathbf{Q}}(\theta), b,-b \mathrm{~N}_{F / \mathbf{Q}}(\theta)>.
$$

Set $\alpha=2 b d_{F}$, so $\beta=b$. Then $\mathrm{N}_{F / \mathbf{Q}}(\beta)=b$ mod squares. We get

$$
q_{(K, \sigma, \alpha)} \oplus<-\mathrm{N}_{F / \mathbf{Q}}(\theta)>\simeq(2 n+1) .<1>,
$$

as claimed.

The following proposition shows that $2 n+3$ is the best possible bound in the case where $n \equiv 1(\bmod 4)$, provided the characteristic polynomial of the matrix is supposed to be separable.

Proposition 3.7. Suppose that $n \equiv 1(\bmod 4)$. If $\sqrt{\theta}$ is an eigenvalue of a skewsymmetric matrix of dimension $2 n+2$ with separable characteristic polynomial, then $-\mathrm{N}_{F / \mathbf{Q}}(\theta)$ is a sum of three squares in $\mathbf{Q}$.

Proof. If $\sqrt{\theta}$ is an eigenvalue of a skew-symmetric matrix of dimension $2 n+2$, then its characteristic polynomial is $P(X)=\left(X^{2}+d\right) f(X)$ for some $d \in \mathbf{Q}$. Suppose that the polynomial $P$ is separable. Apply prop 1.2 . with $P(X)=\left(X^{2}+d\right) f(X)$. Set $A=\mathbf{Q}[X] /(P)$, and let $\tau: A \rightarrow A$ be induced by $X \mapsto-X$. If $(A, \tau)$ is adjoint to some symmetric bilinear form $q$, then $q \simeq q_{(K, \sigma, \alpha)} \oplus<2 a, 2 a d>$ for some $\alpha \in F^{*}, a \in \mathbf{Q}^{*}$. If such a form is isomorphic to the unit form, then $\alpha$ is 
totally positive, $a$ is positive and $d=-\mathrm{N}_{F / \mathbf{Q}}(\theta)$. Using the same argument as in the proof of prop. 3.6., we get

$$
\begin{aligned}
< & \mathrm{N}_{F / \mathbf{Q}}\left(2 \alpha d_{F}\right),-\mathrm{N}_{F / \mathbf{Q}}\left(2 \alpha d_{F}\right) \mathrm{N}_{F / \mathbf{Q}}(\theta)>\oplus<2 a,-2 a \mathrm{~N}_{F / \mathbf{Q}}(\theta)> \\
& \simeq<1,1,1,1>.
\end{aligned}
$$

Multiplying this relation by $2 a$, using lemma 3.4., and simplifying by $<1>$, we get that $-\mathrm{N}_{F / \mathbf{Q}}(\theta)$ is a sum of three squares.

We now deal with the case where $n$ is even.

Theorem 3.8. Let $K$ be a $C M$-field of degree $2 n$, $n$ even. Then $K$ is generated by an eigenvalue of a skew-symmetric matrix of dimension $2 n+4$.

Proof. We first prove that there exist two negative rational numbers $a, b$ such that $w_{2}\left(q_{(K, \sigma, 1)}\right)=\left(-\mathrm{N}_{F / \mathbf{Q}}(\theta),-1\right)+(a, b)$. Since $q_{(K, \sigma, 1)}$ has dimension and signature $2 n$, over $\mathbf{R}$ it is isomorphic to $2 n$. $<1>$. Hence over $\mathbf{R}$, its Hasse-Witt invariant is trivial. Recall now that the elements of $\mathrm{Br}_{2}(\mathbf{Q})$ are quaternion algebras. Hence $\left(-\mathrm{N}_{F / \mathbf{Q}}(\theta),-1\right)+w_{2}\left(q_{(K, \sigma, 1)}\right)=(a, b)$ for some $a, b \in \mathbf{Q}$. Since $\theta$ is totally negative and $n$ is even, we have $\mathrm{N}_{F / \mathbf{Q}}(\theta)>0$. The above relation then shows that $a$ and $b$ are negative.

Set

$$
q=(2 n-3) .<1>\oplus<-\mathrm{N}_{F / \mathbf{Q}}(\theta) a,-\mathrm{N}_{F / \mathbf{Q}}(\theta) b, \mathrm{~N}_{F / \mathbf{Q}}(\theta) a b>.
$$

Let us show that $q \simeq q_{(K, \sigma, 1)}$. It suffices to prove that these two forms have equal dimensions, discriminants, signatures and Hasse-Witt invariants. We have $\operatorname{dim}(q)=2 n$ and $\operatorname{det}(q)=\mathrm{N}_{F / \mathbf{Q}}(\theta)$. Since $\mathrm{N}_{F / \mathbf{Q}}(\theta)>0$, we have $\operatorname{sign}(q)=$ $2 n$. Moreover, $w_{2}(q)=w_{2}\left(<-\mathrm{N}_{F / Q}(\theta)>\otimes<a, b,-a b>\right)=w_{2}(<$ $a, b,-a b>)+\left(-\mathrm{N}_{F / \mathbf{Q}}(\theta),-1\right)=(a, b)+\left(-\mathrm{N}_{F / \mathbf{Q}}(\theta),-1\right)$. Therefore $q$ and $q_{(K, \sigma, 1)}$ have equal invariants, hence they are isomorphic.

Set $\phi=<\mathrm{N}_{F / \mathbf{Q}}(\theta),-a,-b, a b>$. Then we have

$$
\phi \oplus q_{(K, \sigma, 1)} \simeq(2 n-4) .<1>\oplus<<-a,-b, \mathrm{~N}_{F / \mathbf{Q}}(\theta)>>.
$$

The Hasse-Witt invariant of a 3-fold Pfister form is trivial. Moreover, the Pfister form $<<-a,-b, \mathrm{~N}_{F / \mathbf{Q}}(\theta)>>$ has dimension 8 , trivial discriminant and signature 8 , so it is isomorphic to the 8-dimensional unit form. Hence we get $\phi \oplus q_{(K, \sigma, 1)} \simeq(2 n+4) .<1>$. By [3], th. 1, there exists an algebraic number field $L$ with a $\mathbf{Q}$-linear involution $\tau$ and a $\beta \in L$, such that $\phi \simeq q_{(L, \tau, \beta)}$. The proof shows that $L$ is generated by an element $\rho$ with an even irreducible polynomial $g$, and such that $\tau(\rho)=-\rho$. Moreover, there are infinitely many choices for $L$, hence we can assume that $f \neq g$. Applying prop. 1.2. with $P=f g$ gives the desired result. 


\section{References}

[1] Bayer-Fluckiger, E.: Ideal lattices. Proceedings of the Conference Number Theory and Diophantine Geometry: Baker 60, Zürich (1999), Cambridge University Press, 2002, pp. 168-184

[2] Bender, E.A.: Characteristic polynomials of symmetric matrices. Pacific J. Math. 25, 433-441 (1968)

[3] Berhuy, G.: On hermitian trace forms over hilbertian fields. Math. Z. 217, 561-570 (2001)

[4] Cohen, S.D., Odoni, R.W.K.: Galois groups associated with CM-fields, skew-symmetric matrices and orthogonal matrices. Glasgow Math. J. 32, 35-46 (1990)

[5] Krüskemper, M.: On the scaled trace forms and the transfer of a number field extension. J. Number Th. 40, 105-119 (1992)

[6] Scharlau, W.: Quadratic and Hermitian Forms, Grundlehren Math. Wiss. 270, Springer Verlag, 1985 